\title{
Análise mecânica após reforço femoral proximal com polimetilmetacrilato em orifícios duplos alternados*
}

\section{Mechanical Analysis after Proximal Femoral Reinforcement with Polymethylmethacrylate in Alternated Double Holes}

\author{
Anderson Freitas ${ }^{10}$ Mariana de Medeiros Lessa ${ }^{2}$ \\ Pedro Félix Pereira de Oliveira ${ }^{3}$ \\ 1 Instituto de Pesquisa e Ensino, Hospital Ortopédico e Medicina \\ Especializada (IPE-HOME-DF), Brasília, DF, Brasil \\ 2 Serviço de Ortopedia e Traumatologia, Hospital Regional do Gama \\ (HRG-DF), Brasília, DF, Brasil \\ 3 Programa de Residência em Ortopedia e Traumatologia, Hospital \\ Estadual de Sapopemba (HESAP), São Paulo, SP, Brasil \\ ${ }^{4}$ Coordenador do Programa de Residência Médica em Ortopedia e \\ Traumatologia, Hospital Municipal Miguel Couto (HMMC-RJ), Rio de \\ Janeiro, RJ, Brasil
}

Saulo Pereira de Oliveira ${ }^{2(1)}$

\section{Antônio Carlos Shimano ${ }^{5}$}

\begin{abstract}
Endereço para correspondência Anderson Freitas, Instituto de Pesquisa e Ensino do Hospital Ortopédico e Medicina Especializada (IPE-HOME-DF), SHCS 613, Conjunto C, Brasília, DF, 70200-730, Brasil (e-mail: andfreitas28@gmail.com).
\end{abstract}

\begin{abstract}
${ }^{5}$ Departamento de Biomecânica, Medicina e Reabilitação do Aparelho Locomotor, Faculdade de Medicina de Ribeirão Preto, Universidade de São Paulo (FMRP/USP), Ribeirão Preto, SP, Brasil
\end{abstract}

Rev Bras Ortop 2021;56(5):641-646.

\section{Resumo}

Palavras-chave

- fraturas do quadril

- fraturas do fêmur

- osteoporose

- polimetilmetacrilato
Objetivo Avaliar, por meio de ensaio biomecânico, a carga máxima, a energia, e o deslocamento necessários para a ocorrência de fratura em modelos sintéticos de fêmures após a retirada de parafusos acanulados e a realização de técnica de reforço com polimetilmetacrilato (PMMA) em diferentes posições combinadas.

Métodos Foram utilizados 25 ossos sintéticos divididos em 4 grupos: o grupo controle (GC), com 10 modelos sem perfuração, e os grupos teste (A, B e C), com 5 modelos cada. Os grupos de teste foram fixados com parafusos acanulados pela técnica de Asnis, e tiveram a síntese removida e o preenchimento de dois dos orifícios formados por técnica de reforço com PMMA. A análise biomecânica foi realizada simulando queda sobre o grande trocânter utilizando máquina servo-hidráulica.

Resultados Todos os corpos de prova do GC e dos grupos A, B e C apresentaram fratura baso-cervical do colo femoral, exceto um modelo do grupo $B$, que apresentou fratura longitudinal. Foi utilizada uma média de 5,4 mL de PMMA no reforço dos grupos com preenchimento. Segundo a análise de variância (analysis of variance, ANOVA) e o teste de comparações múltiplas de Tukey, no nível de 5\%, observou-se que o GC apresentou diferença significativa em relação aos grupos $A$ e $C$ nos seguintes parâmetros: carga máxima, energia até a fratura, e deslocamento.
Estudo desenvolvido pelo Serviço de Ortopedia e Traumatologia do Hospital Regional do Gama, e pelo Instituto de Pesquisa e Ensino do Hospital Ortopédico e Medicina Especializada (IPE-HOME), Brasília, DF, Brasil. recebido

24 de Fevereiro de 2020

aceito

15 de Abril de 2020

Publicado on-line

Setembro 25, 2021
DOI https://doi.org/

10.1055/s-0040-1714221. ISSN 0102-3616. (c) 2021. Sociedade Brasileira de Ortopedia e Traumatologia. All rights reserved.

This is an open access article published by Thieme under the terms of the Creative Commons Attribution-NonDerivative-NonCommercial-License, permitting copying and reproduction so long as the original work is given appropriate credit. Contents may not be used for commercial purposes, or adapted, remixed, transformed or built upon. (https://creativecommons.org/ licenses/by-nc-nd/4.0/)

Thieme Revinter Publicações Ltda., Rua do Matoso 170, Rio de Janeiro, RJ, CEP 20270-135, Brazil 


\section{Abstract}

\author{
Keywords \\ - hip fractures \\ - femoral fractures \\ - osteoporosis \\ - polymethyl- \\ methacrylate
}

Conclusão Observou-se que os grupos A e C, quando comparados ao GC, apresentaram diferenças significativas na observação do deslocamento, da carga máxima, e da energia até a fratura.

Objective To evaluate, through a biomechanical assay, the maximum load, energy, and displacement necessary for the occurrence of fractures in synthetic models of femurs after the removal of cannulated screws and the performance of a reinforcement technique with polymethylmethacrylate (PMMA) in different combined positions.

Methods In total, 25 synthetic bones were used, and they were divided into 4 groups: the control group (CG), with 10 models without perforation, and the test groups (A, B and $C$ ), with 5 models each. The test groups were fixed with cannulated screws using the Asnis technique, and they had the synthesis removed, and two of the holes formed by the reinforcement technique with PMMA were filled. The biomechanical analysis was performed simulating a fall on the large trochanter using a servo-hydraulic machine. Results All specimens of the CG and of groups A, B and C presented basal-cervical fracture of the femoral neck, except for a single model in group $B$, which presented a longitudinal fracture. An average of $5.4 \mathrm{~mL}$ of PMMA were used to reinforce the groups with filling. According to the analysis of variance (ANOVA) and the Tukey multiple comparison test, at the level of $5 \%$, we observed that the CG presented significant differences in relation to groups $A$ and $C$ in the following parameters: maximum load, energy up to the fracture, and displacement.

Conclusion We observed that groups A and C, when compared to the CG, showed significant differences in the observation of displacement, maximum load, and energy until the fracture.

\section{Introdução}

O aumento exponencial de idosos no mundo gera uma mudança no padrão de morbimortalidade e no enfrentamento de patologias comuns nesta faixa etária, como é o caso da osteoporose. A baixa densidade óssea, a deterioração da microarquitetura, e o aumento da fragilidade podem ter como consequência a diminuição da resistência mecânica deste tecido, predispondo a fraturas por traumas de baixa energia, sendo a fratura do extremo proximal do fêmur (EPF) a que tem o maior índice de morbimortalidade. ${ }^{1-3}$

O tratamento cirúrgico dessas fraturas visa dar ao paciente condições de retorno às atividades. Existem diversos tratamentos preconizados, a exemplo de tutores intramedulares, parafusos acanulados (PAs), e/ou placa de tubo deslizante (PTD). Há ainda a possibilidade de substituição articular por artroplastia do quadril, a fim de diminuir a chance de complicações clínicas devido à imobilidade do paciente. ${ }^{4}$

É comum no uso das sínteses a migração, e, com isso, a persistência de dor na região glútea e na coxa pela proeminência das sínteses, ${ }^{5}$ sendo essa uma das indicações da sua retirada, assim como falha de implante ou infecção. A remoção de implantes pode causar maior fragilidade local e, com isso, possíveis fraturas do EPF, principalmente em pacientes com baixa qualidade óssea., 3,7

Com isso, avaliar a carga máxima, a energia, e o deslocamento necessários para a ocorrência de fratura em modelos sintéticos de fêmures, após retirada dos PAs e realização da técnica de reforço com polimetilmetacrilato (PMMA) em diferentes posições combinadas, por meio de um ensaio estático de flexão simulando queda sobre o trocânter, pode propiciar resultados que determinem o desenvolvimento de técnica alternativa na solução de casos em que seja necessária a remoção das sínteses.

\section{Material e Métodos}

Foram utilizados 25 modelos sintéticos c1010(Nacional Ossos, Jaú, SP, Brasil), fabricados em poliuretano esponjoso e cortical com 10 libras por pé cúbico (pounds per cubic foot, $\mathrm{lb} / \mathrm{ft}^{3}$ ), com canal medular de $12 \mathrm{~mm}$, de mesmo lote e mesmo modelo, e divididos em 4 grupos: grupo controle (GC) com 10 modelos, e grupos teste A, B e C, cada um com 5 modelos.

O GC foi constituído por modelos sintéticos com integridade externa e interna intactas. Os modelos dos grupos A, B e C, sem a realização de fraturas prévias, foram fixados conforme a técnica para PA tipo Asnis (em triângulo invertido): foram submetidos à introdução de fio guia com o auxílio de controle radioscópico, no formato de pares equidistantes entre si, até a distância de $5 \mathrm{~mm}$ da superfície da cabeça femoral. A medição desses fios foi realizada com medidor padrão do fabricante nacional (Ortosintese, São Paulo, SP, Brasil), determinando-se o comprimento dos parafusos tamanho $95 \mathrm{~mm}$. Os pertuitos foram realizados utilizando uma broca acanulada própria do fabricante para a utilização de PAs de $7,5 \mathrm{~mm}$. Foram introduzidos os PAs de 7,5 mm em cada pertuito, com comprimento 


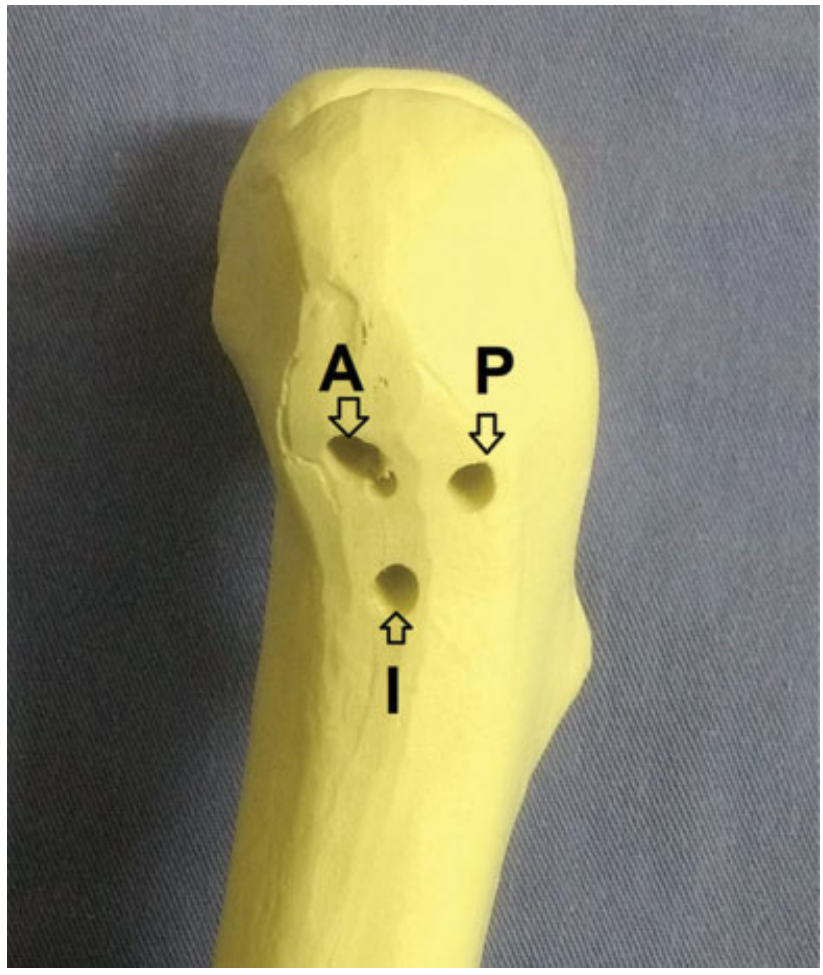

Fig. 1 Exemplar de modelo sintético após retirada do material de síntese, evidenciando os orifícios formados (A: orifício anterior; P: orifício posterior; I: orifício inferior).

previamente determinado pela medida adquirida (95 $\mathrm{mm})$, e, em seguida, foi realizada a retirada deles ( - Figura $\mathbf{1}$ ).

Após a retirada dos implantes dos grupos A, B e C, os modelos sintéticos foram submetidos a uma técnica de reforço com o uso de cimento ósseo tipo PMMA, do fabricante Biomecânica (Jaú, SP, Brasil), de viscosidade normal, preenchendo o pertuito de dois PAs em cada osso, que foi introduzido de forma anterógrada, com o auxílio de uma seringa de $20 \mathrm{~mL}$, e, por meio desta, foi calculado o volume utilizado de PMMA. No grupo A, o preenchimento se deu nos furos das posições anterior e inferior; no grupo B, nas posições anterior e posterior; e no grupo $C$, nas posições posterior e inferior (-Figura 2).

Todas as amostras dos 4 grupos foram submetidas a ensaios estáticos de flexão, utilizando a máquina servohidráulica do modelo MTS 810-FlexTest 40 (MTS Systems, Eden Prairie, MN, EUA), com capacidade de 100 kN. Cada fêmur foi fixado ao dispositivo de ensaios deixando $150 \mathrm{~mm}$ do seu comprimento fora do dispositivo de fixação, em direção ao pistão hidráulico, posicionado na base da máquina de ensaios com inclinação de $10^{\circ} \mathrm{com}$ a horizontal e $15^{\circ}$ de rotação interna, aferidos por meio de goniômetro, mantendo o trocânter maior apoiado em um disco de silicone de $8 \mathrm{~cm}$ de diâmetro (-Figura 3). Foi aplicada uma pré-carga de $40 \mathrm{~N}$, e utilizada uma velocidade de $2 \mathrm{~mm} / \mathrm{s}$ de deslocamento do pistão direcionando na cabeça do fêmur até a fratura (-Figura 3). Os valores de carga máxima foram mensurados em Newtons (N); a energia até a fratura, em Joules (J); e o deslocamento, em milímetros ( $\mathrm{mm}$ ).

Os resultados foram obtidos por meio de uma análise inferencial, composta pela análise de variância para um fator (one-way analysis of variance, one-way ANOVA) juntamente com o teste de comparações múltiplas de Tukey, com o objetivo de verificar se existia diferença de carga máxima, deslocamento e energia até a fratura entre os quatro grupos. O critério de determinação de significância foi o nível de $5 \%$. A análise estatística foi processada pelo software estatístico Statistical Package for the Social Sciences (SPSS, IBM Corp., Armonk, NY, EUA), versão 20.0.

\section{Resultados}

Todos os corpos de prova do GC e dos grupos A e C apresentaram fratura baso-cervical do colo femoral (-Figura 4). No

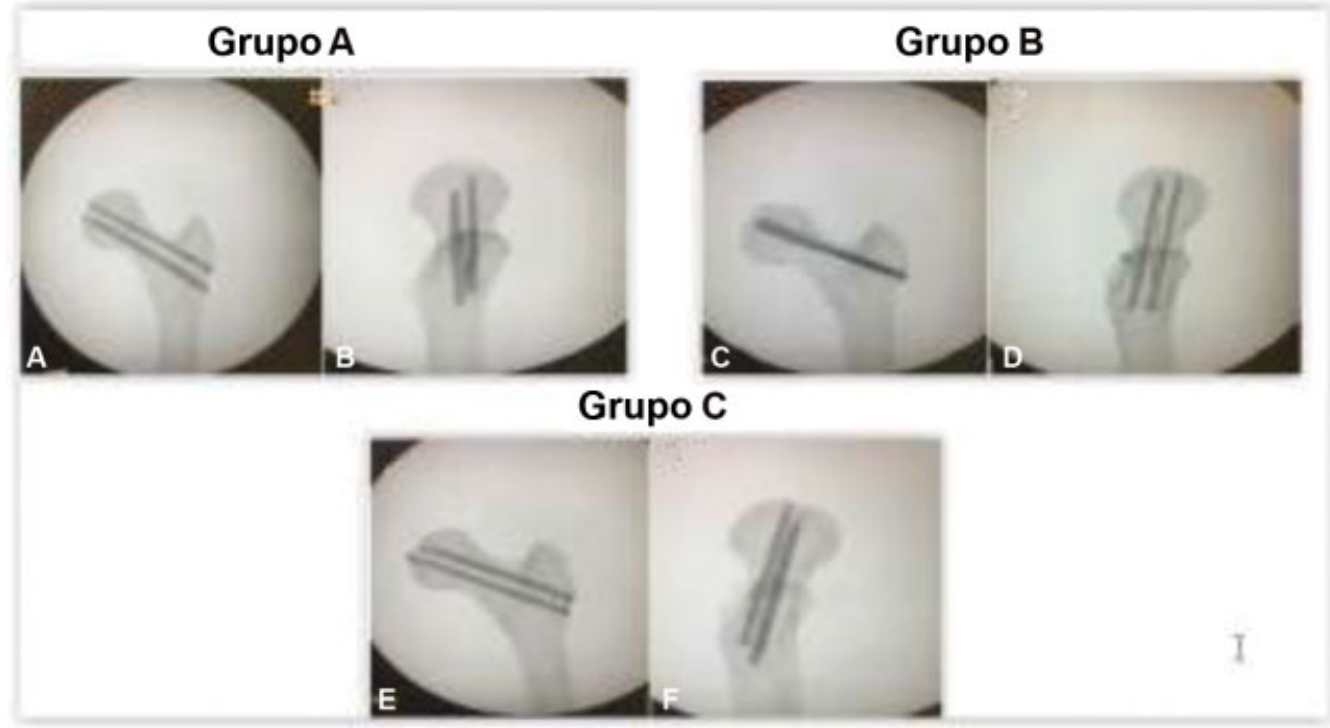

Fig. 2 Imagens fluoroscópicas em incidências anteroposterior e de perfil de modelos dos grupos A, B e C, respectivamente, após o preenchimento de seus orifícios com polimetilmetacrilato (PMMA). (A/B): orifícios anterior e inferior reforçados; (C/D) orifícios anterior e posterior reforçados; (E/F): orifícios posterior e inferior reforçados). 


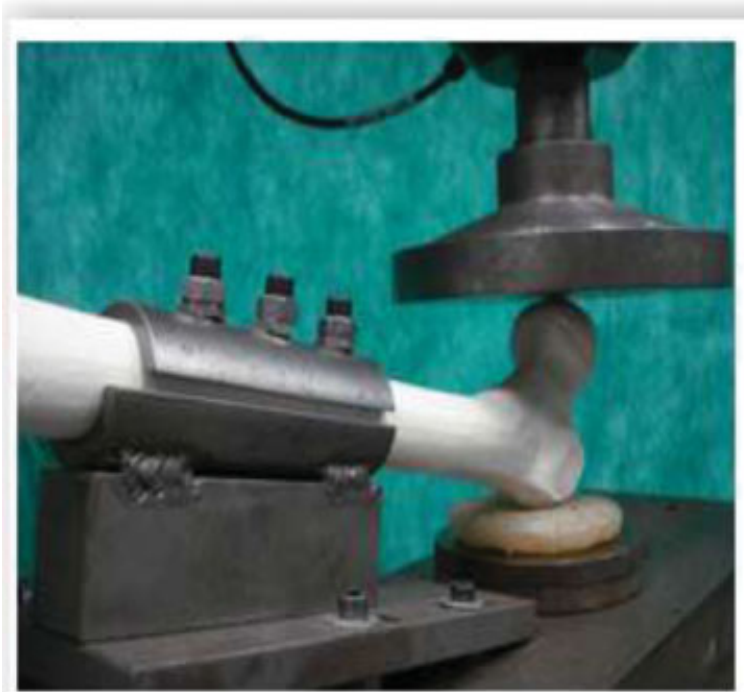

A

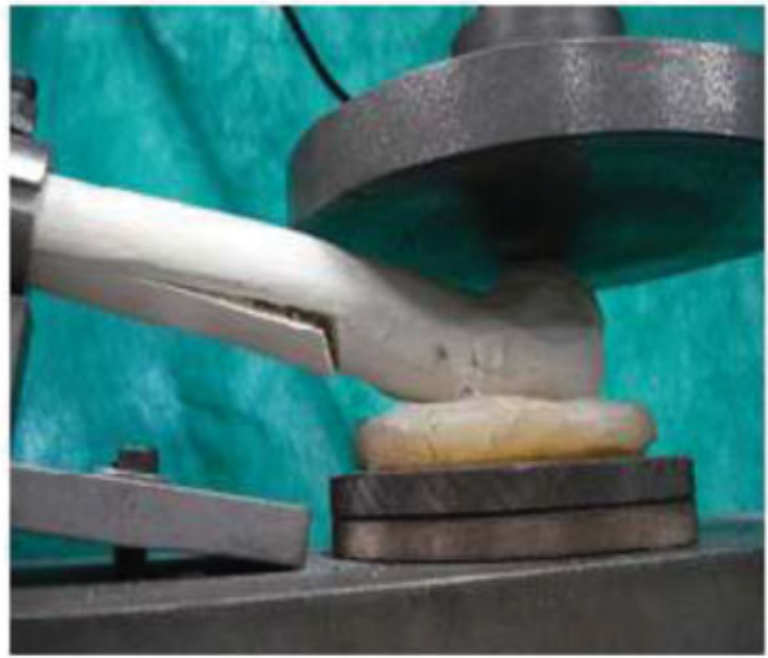

B

Fig. 3 Modelo sintético do grupo B. (A) modelo sintético de fêmur fixado no dispositivo durante o ensaio, demonstrando o posicionamento, e (B) após o ensaio, apresentando fratura longitudinal.

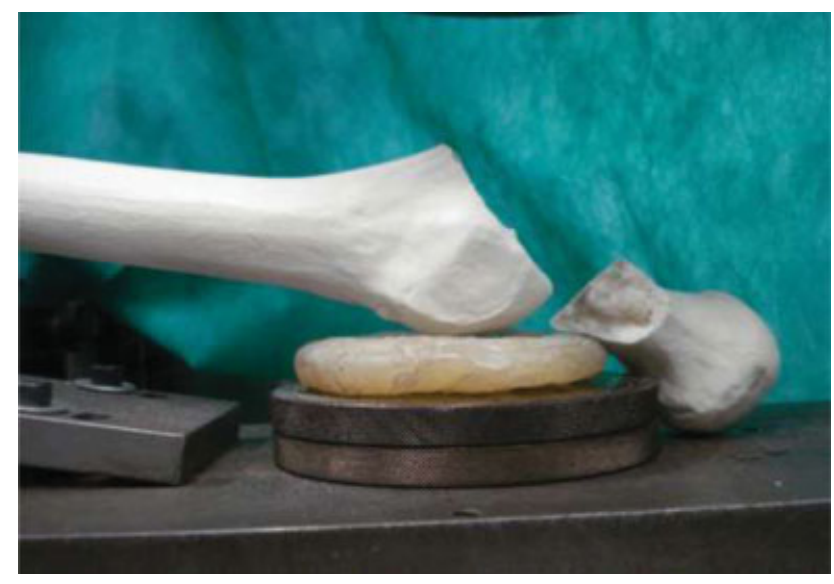

Fig. 4 Modelo sintético de fêmur com fratura baso-cervical.

grupo B, o corpo de prova B1 apresentou fratura longitudinal na região subtrocantérica (-Figura 3B), e todos os demais corpos de prova do grupo $B$ também apresentaram fraturas baso-cervicais do colo femoral.

A quantidade de PMMA utilizada para o preenchimento dos 2 pertuitos dos PAs em cada modelo nos grupos A, B e C apresentou uma média de $5,4 \mathrm{~mL}$.

Os parâmetros analisados no GC e nos grupos A, B e C apresentaram as seguintes médias, e seus respectivos desvios padrão: carga máxima em N $([935] \pm 290 ;[1.320] \pm 160$; $[1229] \pm 264 ;[1.310] \pm 63)$, energia até a fratura em J $([7.0] \pm 2.5 ;[8.6] \pm 2.1 ;[10.2] \pm 3.2 ;[11.0)] \pm 2.1)$, e deslocamento em $\mathrm{mm}([7.7] \pm 1.2 ;[6.4] \pm 0.6 ;[6.7] \pm 1.0 ;[6.7] \pm 0.8)$

(-Tabela 1).

A - Tabela 1, assim como as - Figuras 5, 6 e 7, fornecem uma descrição dos parâmetros de carga máxima, energia até a fratura, e deslocamento, respectivamente, segundo cada grupo, e o correspondente nível descritivo (valor de $p$ ) da one-way ANOVA. O teste de comparações múltiplas de Tukey foi aplicado para identificar quais grupos diferiam significativamente entre si no nível de $5 \%$ (coluna de diferenças significativas da tabela).

Observou-se que o GC, segundo a one-way ANOVA, apresentou diferença significativa em relação aos grupos $\mathrm{A}$ e $\mathrm{C}$ nos seguintes parâmetros: carga máxima $(p=0,012)$, energia até a fratura $(p=0,037)$, e deslocamento $(p=0,082)$.

\section{Discussão}

Existem diversas técnicas descritas para o tratamento de fraturas do colo femoral, que podem variar desde a fixação usando PAs até a realização de artroplastia de quadril. A fixação com múltiplos PAs (MPAs) pode variar conforme a quantidade e o posicionamento dos implantes, fatores estes que influem diretamente na estabilidade do conjunto fratura/síntese. É sabido que o uso de três parafusos na conformação de triângulo invertido é mais estável; porém, o uso de dois parafusos pode ser suficiente para alguns tipos de fratura (estáveis). Desta forma, a importância do que se descreve aqui vem da grande incidência do uso do PA no tratamento da fratura do EPF.?

A fragilidade óssea no pertuito do implante após a retirada se mostrou um fator de risco para fraturas do fêmur proximal. ${ }^{8,9}$ Com isso, se reserva a remoção do material a pacientes selecionados, ${ }^{5}$ ratificando, assim, a importância de descrições de estudos experimentais que demonstram o comportamento mecânico dessa região após a retirada da síntese.

Um trabalho com metodologia semelhante, porém comparando modelos sintéticos com e sem preenchimento após a retirada de PAs na posição de triângulo invertido, realizado por Anderson et al. ${ }^{10} \mathrm{em} \mathrm{2019}$, descreve resultados estatisticamente significativos. No presente estudo, notamos que a tensão proporcionada pelo reforço com PMMA é relevante. 0 perfil de fratura resultante nos leva a crer que a quantidade 
Tabela 1 Média da carga máxima (N), do deslocamento (mm), e da energia até a fratura (J) segundo o grupo de preenchimento

\begin{tabular}{|c|c|c|c|c|c|c|c|}
\hline Variável & $\mathbf{n}$ & média & IC95\% para a média & Mínimo & máximo & Valor de $p^{\mathrm{a}}$ & Diferenças significativas $^{b}$ \\
\hline \multicolumn{8}{|c|}{ Carga máxima $(\mathrm{N})$} \\
\hline Grupo controle & 10 & 935 & $755-1.115$ & 555 & 1399 & \multirow[t]{4}{*}{0,012} & \multirow{4}{*}{$\begin{array}{l}\text { Grupo controle } \neq \\
\text { grupos A e C }\end{array}$} \\
\hline Grupo A & 5 & 1.320 & $1.180-1.460$ & 1.120 & 1.566 & & \\
\hline Grupo B & 5 & 1.229 & $998-1.460$ & 1.063 & 1.691 & & \\
\hline Grupo C & 5 & 1.310 & $1.256-1.365$ & 1.241 & 1.370 & & \\
\hline \multicolumn{8}{|c|}{ Deslocamento (mm) } \\
\hline Grupo controle & 10 & 7,71 & $6,9-8,4$ & 5,3 & 9,5 & \multirow[t]{4}{*}{0,082} & \multirow{4}{*}{$\begin{array}{l}\text { Tendências: grupo } \\
\text { controle } \neq \text { grupo A }\end{array}$} \\
\hline Grupo A & 5 & 6,42 & $5,9-6,9$ & 5,8 & 7,0 & & \\
\hline Grupo B & 5 & 6,76 & $5,9-7,6$ & 5,5 & 7,8 & & \\
\hline Grupo C & 5 & 6,70 & $6,0-7,4$ & 5,8 & 7,6 & & \\
\hline \multicolumn{8}{|c|}{ Energia até a fratura (J) } \\
\hline Grupo controle & 10 & 7,05 & $5,5-8,6$ & 4,4 & 10,4 & \multirow[t]{4}{*}{0,037} & \multirow{4}{*}{$\begin{array}{l}\text { Grupo controle } \neq \\
\text { grupo } C\end{array}$} \\
\hline Grupo A & 5 & 8,60 & $6,7-10,5$ & 6,5 & 11,6 & & \\
\hline Grupo B & 5 & 10,2 & $7,4-13,0$ & 6,0 & 14,3 & & \\
\hline Grupo C & 5 & 10,9 & $9,1-12,8$ & 8,6 & 14,0 & & \\
\hline
\end{tabular}

Notas: análise de variância para um fator (one-way analysis of variance, one-way ANOVA). ' Diferenças significativas, no nível de $5 \%$, segundo o teste de comparações múltiplas de Tukey.

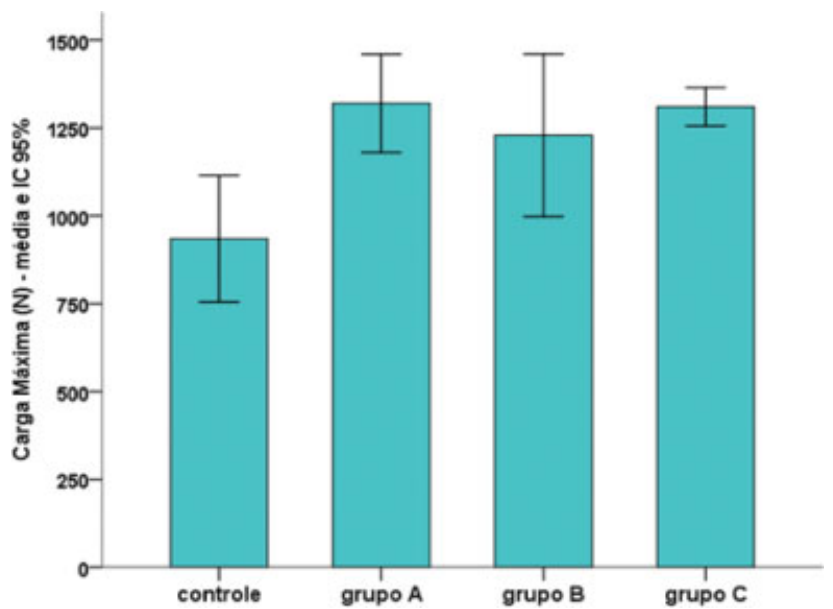

Fig. 5 Carga máxima (N) segundo o grupo de preenchimento.

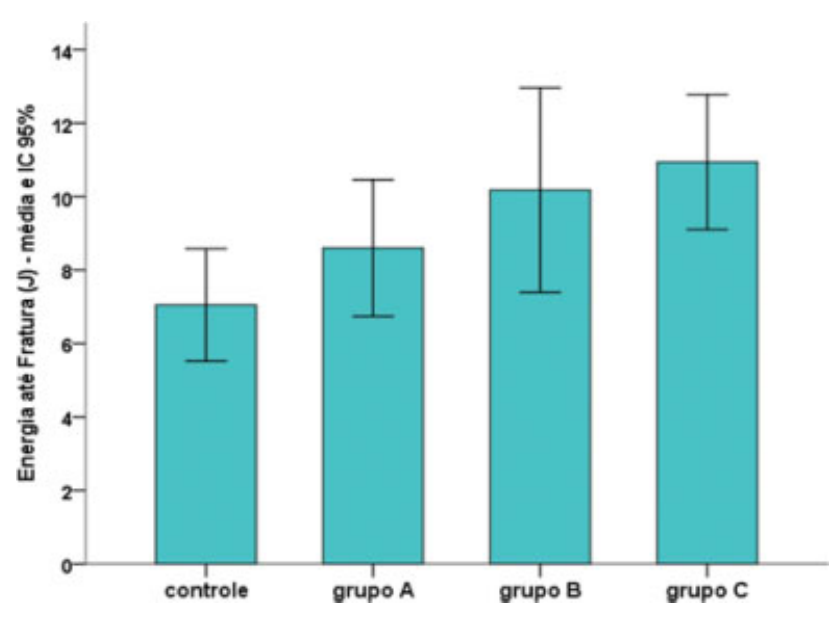

Fig. 6 Energia até fratura (J) segundo o grupo de preenchimento.

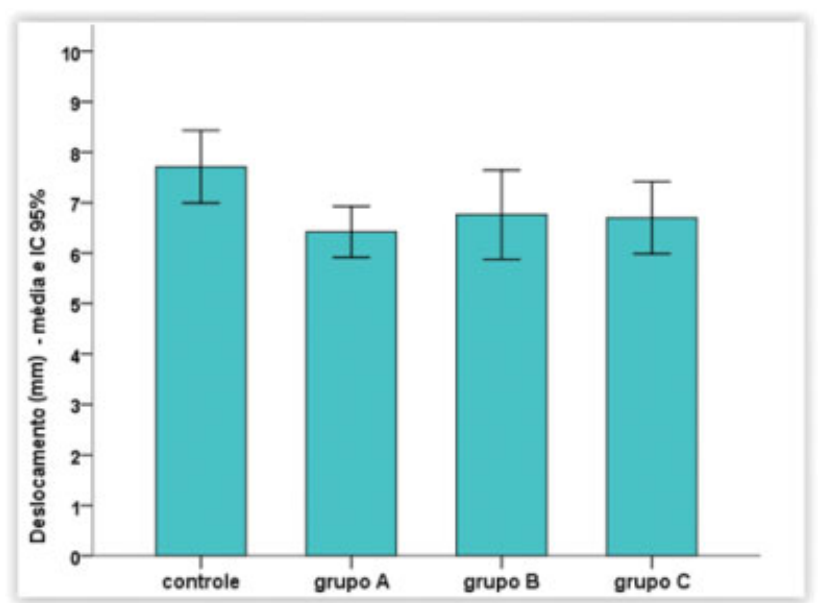

Fig. 7 Deslocamento $(\mathrm{mm})$ segundo o grupo de preenchimento.

de pertuitos preenchidos e o local do reforço são mais importantes do que a quantidade de PMMA utilizada para a técnica. Tal resultado corrobora os achados do estudo citado anteriormente. $^{10}$

As diferenças biomecânica e estrutural entre os ossos sintéticos e os ossos de cadáveres não permitem a comparação dos valores absolutos entre os desenvolvimentos científicos. Apesar disso, há uma equidade ao notarmos o benefício do reforço ósseo com o uso do PMMA. ${ }^{11-13} \mathrm{O}$ uso de reforço ósseo de PMMA após a retirada de implantes já apresenta resultados experimentais. ${ }^{11,14,15}$

Há de se considerar ainda como um possível viés na condição clínica que, com a consolidação, há uma diminuição do comprimento real do eixo longo do colo femoral, o que torna o braço de alavanca menor e pode, eventualmente, aumentar a carga e a energia necessárias para uma nova fratura. 
É possível que a observação de fraturas em um único modelo do grupo B tenha ocorrido por alterações estruturais inerentes à fabricação, uma vez que a fratura se comportou de forma atípica, incomum em situações clínicas com ossos esqueleticamente maduros. Há também de se salientar que os grupos que apresentaram parâmetros significativamente positivos ao reforço com PMMA tiveram em comum o preenchimento do orifício inferior, de forma que tal região possa ser um local de necessidade de reforço ao mecanismo estudado em procedimentos relacionados à profilaxia da fratura do EPF.

Há a dificuldade inerente à realização de ensaios experimentais utilizando modelos cadavéricos em nosso país, o que torna quase que obrigatório que, para este tipo de experimento, sejam utilizados modelos sintéticos, fato este que não diminui a sua importância, contanto que sejam desenvolvidos apresentando sempre um GC.

Modelos humanos cadavéricos apresentam heterogeneidade das amostras (em variáveis como densidade óssea e dimensões) que podem comprometer a observação dos parâmetros analisados, quando não submetidos a uma metodologia de escolha padrão que envolva a realização de densitometrias, radiografias, e outros exames de imagem, fato este não necessário em modelos sintéticos. A escolha de tais modelos permitiu padronizar a avaliação metodológica e garantir que as características biomecânicas pudessem ser comparadas entre os grupos.

\section{Conclusão}

Observou-se que os grupos A e C, quando comparados ao GC, apresentaram diferenças significativas na observação do deslocamento $(p=0,082)$, da carga máxima $(p=0,012)$, e da energia até a fratura $(p=0,037)$.

\section{Suporte Financeiro}

Não houve suporte financeiro de fontes públicas, comerciais, ou sem fins lucrativos.

Conflito de Interesses

Os autores declaram não haver conflito de interesses.

\section{Referências}

1 Souza Júnior EA, Ferreira NF, Lopes PE, Torres MS, Baumfeld DS, Andrade MA. Significado e considerações sobre a osteoporose por mulheres com e sem diagnóstico da doença. ABCS Health Sci 2019;44(01):22-27

2 Gullberg B, Johnell O, Kanis JA. World-wide projections for hip fracture. Osteoporos Int 1997;7(05):407-413

3 Yang JH, Jung TG, Honnurappa AR, et al. The Analysis of Biomechanical Properties of Proximal Femur after Implant Removal. Appl Bionics Biomech 2016;2016:4987831

4 March LM, Chamberlain AC, Cameron ID, et al. Fractured Neck of Femur Health Outcomes Project Team. How best to fix a broken hip. Med J Aust 1999;170(10):489-494

5 Tosounidis TH, Castillo R, Kanakaris NK, Giannoudis PV. Common complications in hip fracture surgery: Tips/tricks and solutions to avoid them. Injury 2015;46(Suppl 5):S3-S11

6 Eberle S, Wutte C, Bauer C, von Oldenburg G, Augat P. Should extramedullary fixations for hip fractures be removed after bone union? Clin Biomech (Bristol, Avon) 2011;26(04):410-414

7 Basile R, Pepicelli GR, Takata ET. Osteosynthesis of femoral neck fractures: two or three screws? Rev Bras Ortop 2015;47(02): 165-168

8 Kukla C, Pichl W, Prokesch R, et al. Femoral neck fracture after removal of the standard gamma interlocking nail: a cadaveric study to determine factors influencing the biomechanical properties of the proximal femur. J Biomech 2001;34(12):1519-1526

9 Mahaisavariya B, Sitthiseripratip K, Suwanprateeb J. Finite element study of the proximal femur with retained trochanteric gamma nail and after removal of nail. Injury 2006;37(08): 778-785

10 Anderson F, Ramos LS, Dantas EL. Giordano Neto V, Godinho PF, Shimano AC. Ensaio biomecânico após retirada de parafusos canulados do fêmur proximal (análise in vitro). Rev Bras Ortop 2019;54(04):416-421

11 Beckmann J, Ferguson SJ, Gebauer M, Luering C, Gasser B, Heini P. Femoroplasty-augmentation of the proximal femur with a composite bone cement-feasibility, biomechanical properties and osteosynthesis potential. Med Eng Phys 2007;29(07):755-764

12 Fliri L, Sermon A, Wähnert D, Schmoelz W, Blauth M, Windolf M. Limited V-shaped cement augmentation of the proximal femur to prevent secondary hip fractures. J Biomater Appl 2013;28(01): 136-143

13 Basafa E, Murphy RJ, Otake Y, et al. Subject-specific planning of femoroplasty: an experimental verification study. J Biomech 2015;48(01):59-64

14 Strauss EJ, Pahk B, Kummer FJ, Egol K. Calcium phosphate cement augmentation of the femoral neck defect created after dynamic hip screw removal. J Orthop Trauma 2007;21(05):295-300

15 Heini PF, Franz T, Fankhauser C, Gasser B, Ganz R. Femoroplastyaugmentation of mechanical properties in the osteoporotic proximal femur: a biomechanical investigation of PMMA reinforcement in cadaver bones. Clin Biomech (Bristol, Avon) 2004;19(05): 506-512 